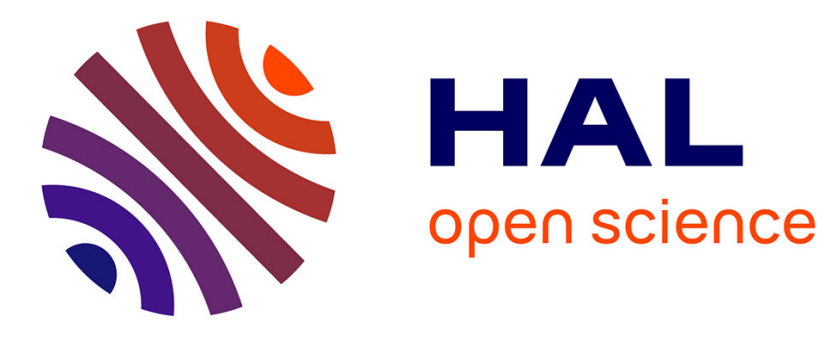

\title{
Recognizing Driver Awareness of Pedestrian
}

\author{
Minh Tien Phan, Vincent Frémont, Indira Thouvenin, Mohamed Sallak, \\ Véronique Cherfaoui
}

\section{To cite this version:}

Minh Tien Phan, Vincent Frémont, Indira Thouvenin, Mohamed Sallak, Véronique Cherfaoui. Recognizing Driver Awareness of Pedestrian. 17th International IEEE Conference on Intelligent Transportation Systems (ITSC 2014), Oct 2014, Qingdao, China. pp.1027-1032. hal-01083967

\section{HAL Id: hal-01083967 https://hal.science/hal-01083967}

Submitted on 18 Nov 2014

HAL is a multi-disciplinary open access archive for the deposit and dissemination of scientific research documents, whether they are published or not. The documents may come from teaching and research institutions in France or abroad, or from public or private research centers.
L'archive ouverte pluridisciplinaire HAL, est destinée au dépôt et à la diffusion de documents scientifiques de niveau recherche, publiés ou non, émanant des établissements d'enseignement et de recherche français ou étrangers, des laboratoires publics ou privés. 


\title{
Recognizing Driver Awareness of Pedestrian
}

\author{
Minh Tien Phan, Vincent Frémont, Indira Thouvenin, Mohamed Sallak and Véronique Cherfaoui
}

\begin{abstract}
In this paper, we propose a novel approach to recognize the awareness or the unawareness that a driver has of a pedestrian appearing on the road in front of the vehicle. Based on the theory of situation awareness and the collected driving data from the on board sensors, a suitable Hidden Markov Model (HMM) is used to model the "Driver Awareness of Pedestrian" and the "Driver Unawareness of Pedestrian". These behaviors are then recognized by using a maximumlikelihood decision method. A real-time validation taken on a driving simulator shows that the model and the output decisions are accurate and efficient.
\end{abstract}

Index Terms-Driver Behaviors Modeling; Pedestrian Safety; Situation Awareness ; Hidden Markov Model;

\section{INTRODUCTION}

$\mathbf{T}$ HE increasing number of pedestrian accidents has become a serious society problem [20]. In order to prevent pedestrians from road accident, several advanced driver assistance systems (ADAS) have been proposed to detect pedestrians using the on-board sensors and to inform the driver of their presences [6]. However, most of these systems do not adapt to the driver's state and can become extremely distracting and annoying when they detect pedestrian. Therefore, taking into account the driver's states and the critical situations are the key features for such a system to work more efficiently.

In this study, we propose an analysis of the cognitive process and the reactions of the driver in a particular situation. The first objective is to observe the driver reactions in the situation in which there is a pedestrian appears in front of the vehicle. The second objective is to provide a non-intrusive detection system that is able to identify whether the driver is aware of a pedestrian (DAP) or whether the driver is unaware of a pedestrian (DUP).

The global recognition system is described in the Fig. 1. Based on different driving-action measurements coming from the vehicle sensors $(1,2)$ and two distinct Hidden Markov Models (HMMs) that are associated with the DAP and the DUP (3), hence, the maximum-likelihood decision method is used to recognize the DAP and the DUP during the driving time (4). In this study, a preliminary experiment is also presented with a driver using a driving simulator. The collected data are used to train and to validate the recognition system. The results show that the system is quite performing with more than $98 \%$ of good recognition.

The paper is organized as follows: Part II presents the previous works that used HMM to model the driver behav-

Phan Minh Tien,Vincent Frémont, Indira Thouvenin, Mohamed Sallak and Véronique Cherfaoui, Heudiasyc Laboratory, University of Technology of Compiegne, France, e-mail: minh.phan@hds.utc.fr.

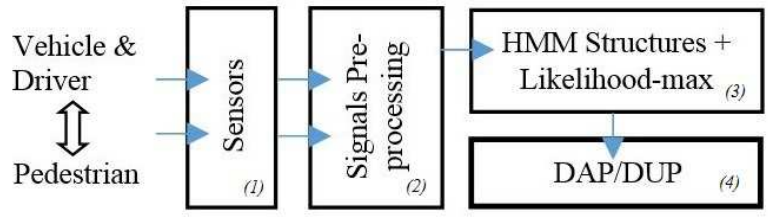

Figure 1. Methodology of DAP/DUP recognition

iors. Part III presents the DAP-DUP modeling. In Part IV, we propose an experiment protocol that allows to collect the data representing the DAP and the DUP. In Part V, we present some numerical results followed by a discussion on the limitations. Finally, the future work and a conclusion are provided in part VI.

\section{RELATED WORK}

Recently, researchers have been investigating drivers behaviors prediction in different context using a looking-in and looking-out framework (LiLo)[19][11][2][12], where sensors simultaneous capture the surrounding environment of a vehicle, its dynamic state through on-board inertial sensors, the internal activities, state of a driver and other cockpit occupants. Nevertheless, most of these works focused on the driver intents and none of them considered the driver awareness of pedestrian. The most closely work is the one from [4]. In this work, the authors used the driver's operational data such as pressure on the accelerator pedal, pressure on the brake pedal (called acceleration reaction) to estimate the driver awareness of a pedestrian. Their hypothesis was that whenever a pedestrian appears on the road, if the driver has noticed it, he had to do an acceleration reaction. Based on a driving behavior dataset collected in natural driving conditions, the authors proposed a probabilistic model in which they calculated the probability of an acceleration reaction is caused in response to driver's awareness of a pedestrian.

However, car driving is a complex activity that involves every levels of human cognition and requires an important level of situation awareness (SA) [1]. Hence, an acceleration reaction is insufficient to confirm the driver awareness of a pedestrian. It is important to model driver states through time and a suitable tool to recognize temporal data patterns is the Hidden Markov Model (HMM). The HMM formulation conveniently decomposes the DUP or the DAP behaviors into distinct atomic levels with a specified duration and incorporates driving actions.

After having been introduced in the late 1960s, Hidden Markov Models (HMMs) have grown popular in a wide range 
of fields, and this encourage us to propose them for the quantification of Driver Awareness of Pedestrian. The HMMs have been successfully used is speech recognition [8]. They were also applied in several pattern recognition areas such as signature recognition [21], gesture recognition [18], etc. In the driving context, Liu and Perlant [10] used HMMs with a dynamical scheme to predict the driver actions (right turn, left turn and stop) within the first 2 seconds of an action sequence. Based on HMM approach, seven types of driving events (right curves, left curves,...) were recognized using only vehicle and acceleration signals as raw data [13]. In [22], a single HMM was used to identify the vehicles in conflict with other vehicles in a limited intersection road with appropriate measurements of the ego-vehicle and surrounding vehicle dynamics. The authors in [5] aimed also to estimate the driving behavior (Left or Right turn, straight or Stop) at intersection from HMM using on the filtered vehicle data.

In [9], a double-layer HMM was built to recognize the driving intention and to predict the driver behaviors. The study used the driving signals such as accelerator pedal position, brake force, steering wheel angle or vehicle speed. The lower layer was a continuous version of the HMM where the observation is considered as a Gaussian distribution, this layer was used to recognize various short-term driving behaviors (brake slowly, accelerate quickly, etc.) in single work case. The upper layer was a discrete HMM that indicated long-term driving intention (emergency braking, obstacle avoidance, etc.) in a combined working case.

Different HMM frameworks have been used in the works above. However, in this study, based on the driving-action data, we propose to build two one-level-discrete HMMs for two distinct behaviors of the driver: Awareness of Pedestrian (DAP) and Unawareness of Pedestrian (DUP). This approach is more suitable to estimate these complex behaviors because it is difficult to characterize the meaning of each short driving action.

\section{DRIVER AWARENESS OR UNAWARENESS OF PEDESTRIAN}

\section{A. Hidden Markov Models}

A Hidden Markov Model (HMM) can be considered as a dynamic Bayesian Network with two concurrent stochastic processes, a Markov process and a general stochastic process [17]. That is, in a HMM, the states are not directly measurable, but the output, dependent on the states, are observable. Different probability parameters give the relation among the states, and between the states and the visible output. A HMM can be characterized by:

- A set of $N$ distinct states $S=\left\{S_{1}, S_{2}, \ldots, S_{N}\right\}$ of system.

- The initial state distribution $\Pi=\left\{\pi_{1}, \pi_{2}, \ldots, \pi_{N}\right\}$ where $\pi_{i}=P\left(s_{t}=S_{i}\right), 1 \leq i \leq N$. Where $s_{t}$ is the state of system at time t.

- The state transitions probability distribution $A=\left\{a_{i j}\right\}$ where $a_{i j}=P\left(s_{t}=S_{j} \mid s_{t-1}=S_{i}\right), 1 \leq i, j, \leq N$.

- Each state can produce one of $M$ distinct observation symbols from the set $V=\left\{V_{1}, V_{2}, \ldots, V_{M}\right\}$.
- The emission probability: $B=\left\{b_{j}(m)\right\}$ where $b_{j}(m)=$ $P\left(v_{t}=V_{m} \mid s_{t}=S_{j}\right), 1 \leq m \leq M, 1 \leq j \leq N$. Where $v_{t}$ is the observation at time t.

- Therefore, the HMM can be written as follows $\lambda=$ $\{A, B, \Pi\}$.

\section{B. HMM-based DAP and DUP Modeling}

We adapt the situation awareness theory [3] to represent the Driver Awareness of Pedestrian (DAP) and the Driver Unawareness of Pedestrian (DUP). The Situation Awareness is defined intuitively as "knowing what is going on". More formally, it is defined as "the perception of the elements in the environment within a volume of time and space (level 1), the comprehension of their meaning (level 2) and the projection of their status in the near future (level 3)" [3]. This happens like a closed loop during the driving time.

In the situation where a pedestrian appears on the road in front of the vehicle, the driver's behaviors could be seen as a sequence of state through Perception (S1) - Comprehension (S2) - Projection (S3). These states are not directly observable but can be characterized by a set of driving actions which is called an observation vector. It could be assumed that the driver will adopt different sequences of action with different levels in each action when he is aware or unaware of a pedestrian.

We considered three temporal signals of driving actions: Accelerator Pedal Position a(t), Braking Force $b(t)$ and Steering Wheel Angle $c(t)$. The observation vector is therefore $\{a(t), b(t), c(t)\}$ that is, a three dimensional continuous vector. In order to simplify the HMMs, the signals of driving actions are discretized in three levels as follows:

The discrete Accelerator pedal position $a_{d}(t)$ is set as:

- [0] Light if $a(t)$ is in $[0,0.1]$

- [1] Medium if $a(t)$ is in ]0.1, 0.5[

- [2] Deep if $a(t)$ is in $[0.5,1]$

The discrete Braking Force $b_{d}(t)$ is set as:

- [0] No Braking if $b(t)$ is equal to 0

- [1] Light Braking if $b(t)$ is in ]0,100[

- [2] Deep Braking if $b(t)$ is in [100, 400]

The discrete Steering Wheel Angle $c_{d}(t)$ is set as:

- [0] Turn Left if $c(t)$ is lower than 0

- [1] Keeping ahead if $c(t)$ is equal to 0

- [2] Turn Right if $c(t)$ is higher than 0

Each dimension of the observation vector $\left\{a_{d}(t), b_{d}(t), c_{d}(t)\right\}$ has therefore three symbols $[0 ; 1$; 2]. This observation vector is then converted into a one dimensional vector $\left\{V_{t}\right\}$ that takes value within $[1 . .27]$ symbols by using: $V_{t}=a_{d}(t) \cdot 3^{2}+b_{d}(t) \cdot 3^{1}+c_{d}(t) \cdot 3^{0}+1$.

Let $\left\{S_{i}\right\}_{i \in(1 . .3)}$ be a discrete, homogenous, Markov chain representing the $N=3$ states of the DAP or the DUP. Finally we have a HMM with 3 hidden states and 27 observation states (Fig. 2).

The DAP and DUP are modeled separately. Indeed, a HMM $\lambda_{D A P}=\left\{A_{D A P}, B_{D A P}, \Pi_{D A P}\right\}$ represents the DAP 


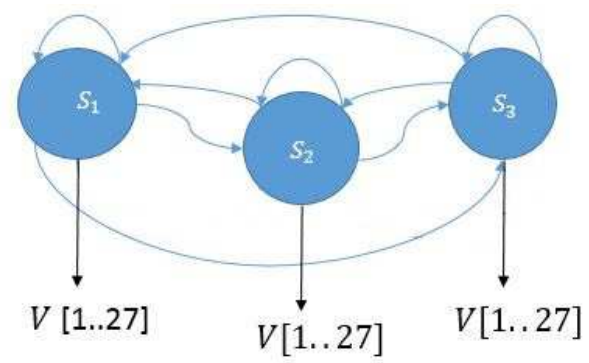

Figure 2. HMM model for DAP or DUP

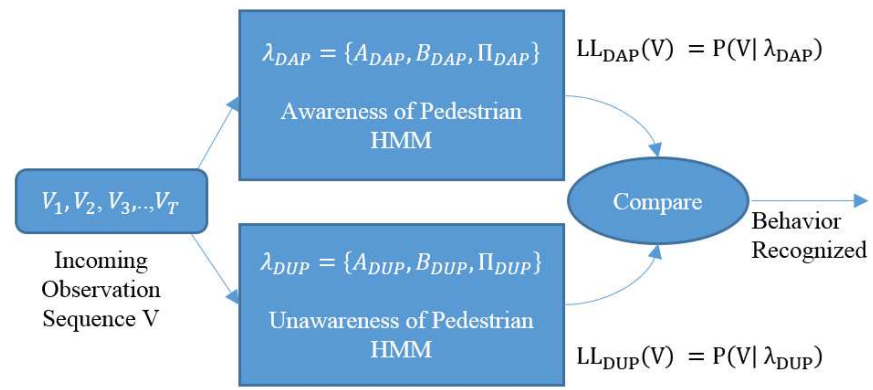

Figure 3. Choosing the behaviors that "best" describes V

and another HMM $\lambda_{D U P}=\left\{A_{D U P}, B_{D U P}, \Pi_{D U P}\right\}$ represents the DUP.

These two HMMs could be trained with annotated data by using standard methods such as the Baum-Welch method and Expectation-Maximization method [17]. In our study, the HMMs are developed by using the Matlab HMM toolbox [14].

\section{DAP and DUP Recognition Process}

In the recognition phase, with each observation sequence extracted and introduced into both DAP HMM and DUP HMM, two likelihoods (LL) are then calculated. The decision is taken by selecting the model which has the higher likelihood (Fig. 3). Indeed, each likelihood represents the probability that the observed sequence would be generated by each model. The likelihood value is calculated by using the forward-backward algorithm [17].

In this section, we have proposed a DAP-DUP model and a decision process to recognize the driver awareness or unawareness of a pedestrian. In order to use this model, a training dataset is needed. In the next section, an experiment is presented including the way to collect and to annotate the data.

\section{EXPERIMENTS DESIGN}

\section{A. Platform}

The experiments are conducted on the driving simulator manufactured by [16]. This simulator is designed to be the most comfortable as possible in order to facilitate various conditions of the experiments. The simulator is configured as shown in the Fig. 4. Three 17 -inches screens are placed

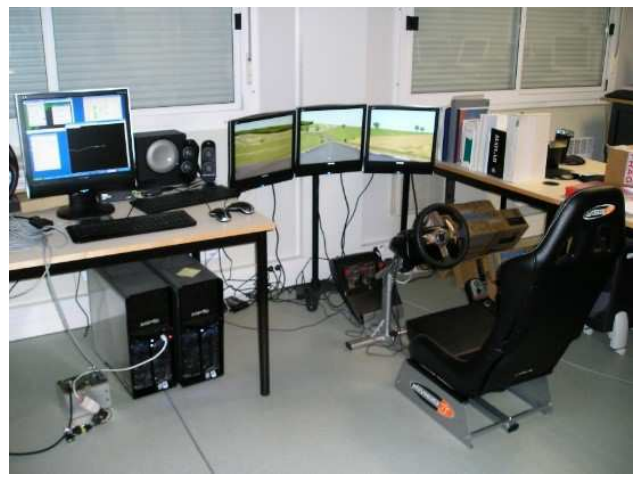

Figure 4. Experimental Platform

at 1.5 meters in front of the driver with a real steering wheel mounted at a real comfortable position near the driver. The simulator is controlled by the driving engine SCANeR-Studio [16] which enables to create different driving scenarios as well as to record all necessary driving signals described above.

\section{B. Scenarios}

In order to limit the complexity of the situations, all scenarios contained no others vehicles and only one pedestrian in each scenario. The ego-vehicle and road parameters such as vehicle weight, size, or others features were fixed to approach real-world conditions. The test track was chosen to be a onelane main road passing through a village. The maximum speed of the vehicle was limited to $80 \mathrm{~km} / \mathrm{h}$ to discourage excessive speeding from the driver.

The Time-To-Collision (TTC) was used to indicate the critical moment that helps to annotate the DAP or the DUP data. Indeed, the TTC is defined as: "The time required for two vehicles to collide if they continue at their present speed and on the same path" [7]. We calculated it by using the vehicle and the pedestrian data given by SCANeR-Studio. $T T C=\frac{\sqrt{\left(x_{v}-x_{p}\right)^{2}+\left(y_{v}-y_{p}\right)^{2}}}{V_{v} \pm V_{p}}$ where $x_{v}, y_{v}, x_{p}, y_{p}$ are the positions of the vehicle and the pedestrian. $V_{v}, V_{p}$ are their speed respectively.

We performed the data acquisition during ten days, in different daytime, with only one driver, 25 years old, who had one year licensed driving and was familiar with the simulator.

The experiment proposes two situations in which the driver was led to be aware or unaware of a pedestrian. We called them the DAP and the DUP simulations.

In the DAP simulation, before each driving, we encouraged the driver to avoid as possible as he could the accident with the pedestrian. The message of TTC value was displayed through the driving time. At $4 s$ of TTC, another message "Warning! Pedestrian!" was displayed to ask the driver to be aware of the pedestrian. In the DUP simulation, the same scenarios as in the DAP simulation with no pedestrian, no message (more exactly, the pedestrian of the DAP simulation is invisible) were also used. The driver was asked to drive normally. In order to annotate the DUP and the DAP data, three hypotheses are considered: 


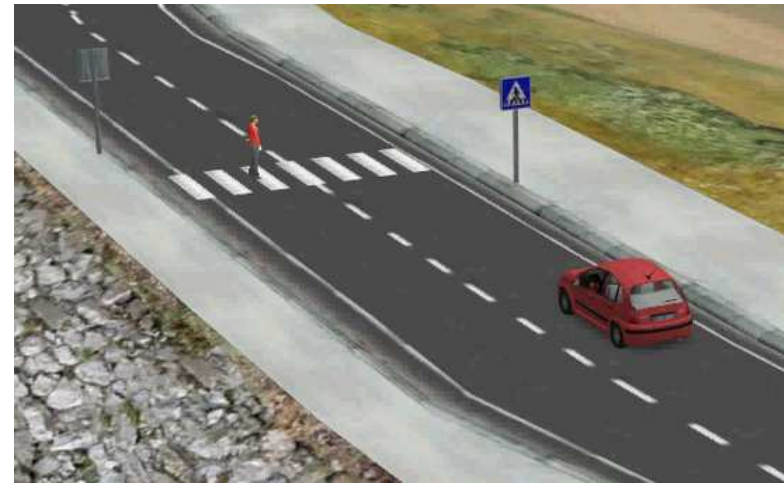

Figure 5. Simulation of Scenario 2, view on scenario-builder mode - The driver can turn the steering wheel and passed by the pedestrian or stop in front of the pedestrian.

1. The driver is aware of a pedestrian when the pedestrian appears clearly on the center screen, and the message "Warning! Pedestrian!" is displayed.

2. The awareness of a pedestrian is a permanent behavior. If the driver is aware of a pedestrian at time $t$, he is considered to be aware of that pedestrian until he passes by the pedestrian or stops in front of the pedestrian.

3. If the driver is unaware of a pedestrian, he drives and does the same maneuvers on the vehicle like there is no pedestrian on the road.

Five scenarios of pedestrian on straight road were proposed and the driver had to drive five times in each scenario, with two DAP and DUP simulations :

Scenario 1: A pedestrian walks along the sidewalk in same direction of the vehicle.

Scenario 2: A pedestrian crosses the road at the crossing mark. A sample is showed in Fig. 5.

Scenario 3: A pedestrian runs on the sidewalk and suddenly crosses the road at the crossing mark.

Scenario 4: A pedestrian runs on the sidewalk .

Scenario 5: A pedestrian crosses the road not at the crossing mark and then crosses the road again not at the crossing mark neither.

\section{Data Extraction}

The driving actions data are automatically logged into hard-disk at $20 \mathrm{~Hz}$ without any filtering or smoothing operations. The vehicle speed is in $\mathrm{km} / \mathrm{h}$. The acceleration pedal position is in $[0 ; 1]$. The brake force is in Newton $(N)$ and takes value in $[0 ; 400]$. The steering wheel angle is in radian $(\mathrm{rad})$. During each driving time, from the instant of $4 s$ of TTC to the instant that the vehicle passes by the pedestrian or stops in front of the pedestrian, we extract all data in this time period. Because of the different driver reactions, each period has different length from $3 s$ to $5 s$ (from 60 to 100 value points)

In the DAP simulations, we can see some reactions of the driver such as braking and stopping in front of the pedestrian or decelerating and turning to avoid the pedestrian and passing by him, etc. For example, in the Fig. 6, the driver
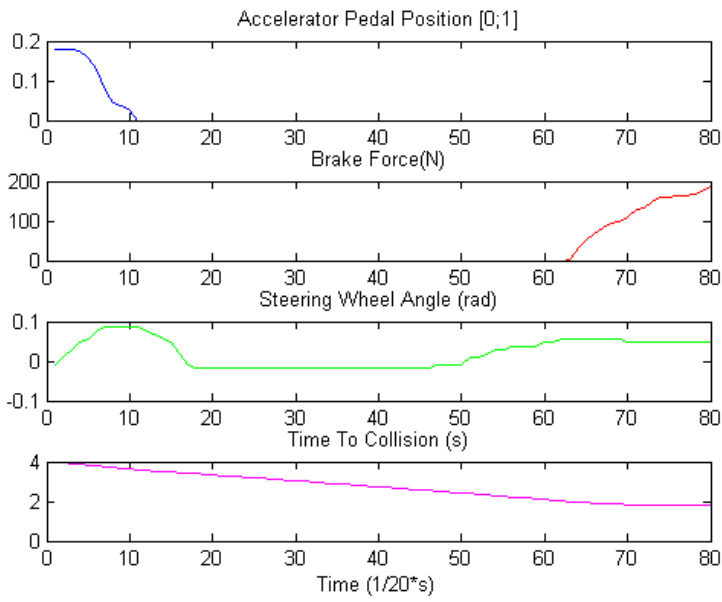

Figure 6. DAP data sample - A deceleration occurs at $4 \mathrm{~s}$ of TTC and a braking occurs at $2 \mathrm{~s}$ of TTC
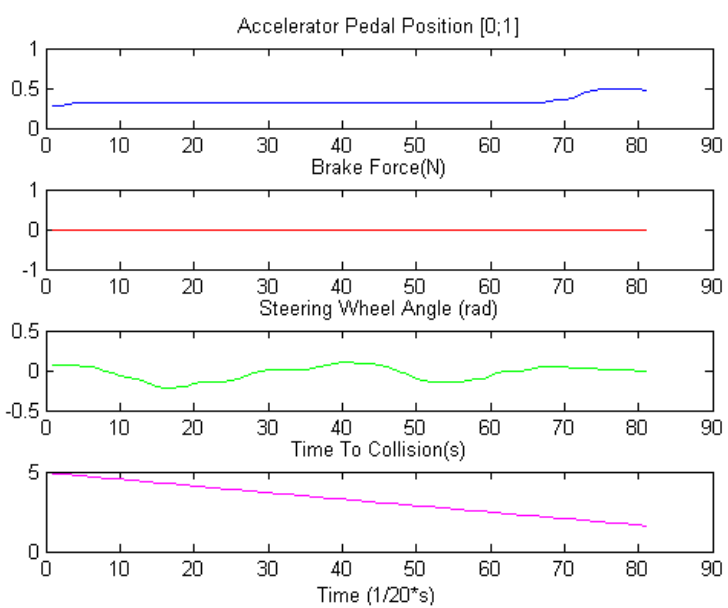

Figure 7. DUP data Sample - None of the deceleration reactions occurs during the critical time

releases accelerator pedal at $4 s$ of TTC and at $2 s$ of TTC, he begins braking. On the other hand, the DUP simulations showed that none of these reactions occurs (Fig. 7).

\section{RESUlTS \& DisCUSSION}

In order to evaluate the recognition system, we define the True Positive Rate (TPR) which is the sum of the number of correct DAP recognized and the number of correct DUP recognized divided by the number of test data. We propose to verify the system using two validations: the mixed-scenarios cross validation and the inter-scenarios validation.

\section{A. Mixed-scenarios Cross Validation}

All data obtained from the different experiments described above are segmented into different small sub-sequences depending on the time step that we suppose to use in the recognition phase. For example, if the chosen time step for DAP/DUP recognition is $2 s$, the data will be then segmented into 1256 sub-sequences of $2 s$ annotated DAP and 989 subsequences of $2 s$ annotated DUP. 


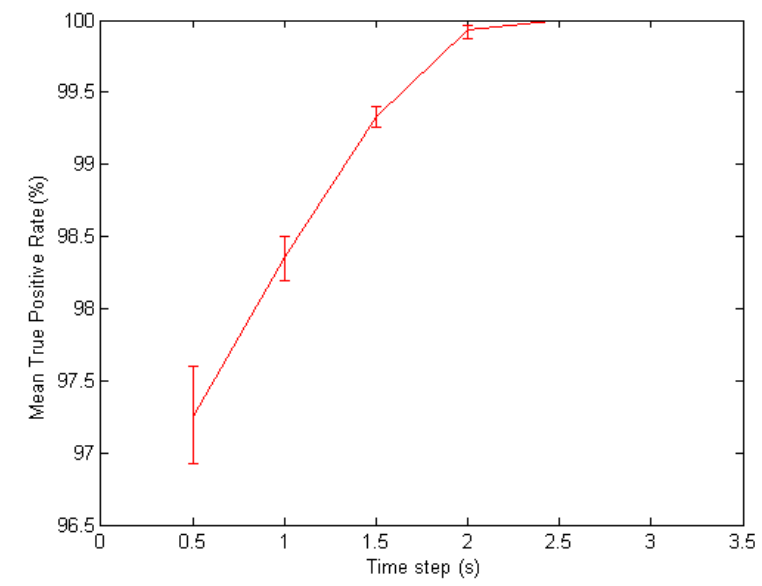

Figure 8. Recognition results for different time-steps

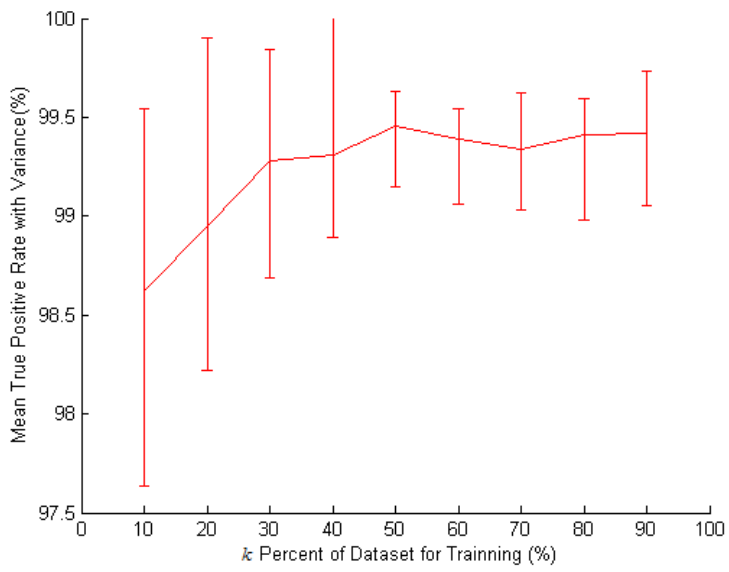

Figure 9. Cross Validation of DAP/DUP recognition

In order to identify the time-step that was the most suitable for recognizing the DAP or the DUP, we mix randomly all DAP sub-sequence data, and mix randomly all DUP subsequence data, we use 50\% of this data for training and $50 \%$ remaining for recognizing, we repeat this process and plot the curve of the mean of TPR in different time-steps (Fig. 8 ). Finally, the time step of $2 s$ (40 data points) is chosen to intercept sensor data and for the recognition because it meets a high TPR (99.7\%) and guarantees a good number of data for training and for testing (1256 sequences for DAP and 989 sequences for DUP).

With a time step of $2 s$, we randomly split the mixed dataset into $k \%$ for training and $(100-k) \%$ for validation. For each split, we train the model with the training data, and the TPRs are assessed using the validation data. We repeat this procedure and plot the curve of this cross-validation. The results are showed in Fig. 9 and highlight that the decision process is performing and stable (up to $99.5 \%$ TPR).

With $k=50 \%$ of the dataset for training and $50 \%$ of the dataset for validation, the DAP HMM converges at $18^{\text {th }}$ iteration and the DUP HMM converges at $26^{\text {th }}$ iteration. Now, let us consider the score of the subtraction between the two likelihoods: $L L(V)=L L_{D A P}(V)-L L_{D U P}(V)$.

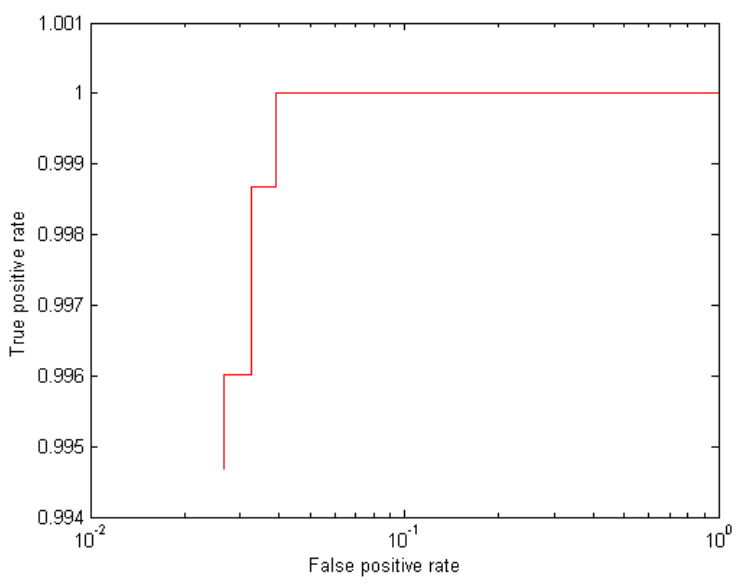

Figure 10. ROC curve - Performance of the decision process

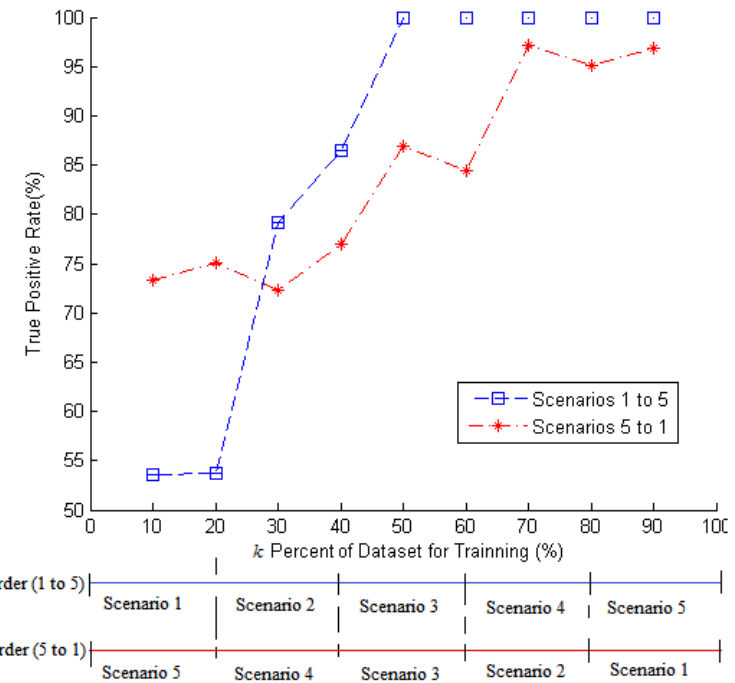

Figure 11. DAP and DUP recognition inter-scenarios validation

The ROC curve related to this score $L L(V)$ indicates the accuracy of the decision process (Fig. 10).

\section{B. Inter-scenarios Validation}

Next, we validate the model with the unmixed data. The sub-sequence data of all scenarios are ordered from scenario 1 to scenario 5 (Fig. 11 square blue line) or from 5 to 1 (Fig. 11 star red line). In this case, $k \%$ of the dataset are fitted for training and $(100-k) \%$ of the dataset for validation. We can see in these curves, the more scenarios are used for training, the better TPR we get. Moreover, with $20 \%$ of the dataset for training $(k=20)$, it means that the both DAP, DUP HMMs are trained with only the data of scenario 1 (square blue line) (or scenarios 5 respectively in star red line) and the decision process is validated with the data of scenario 2 to 5 (scenario 1 to 4 respectively in star red line). The results of the decision process are even good of 54\% TPR $(75 \%$ respectively in star red line). 


\section{Limitations}

In this paper, we have presented a novel approach for recognizing the driver awareness or unawareness of pedestrian. This is a case to describe the coupling of the vehicledriver-environment through the driving actions. The theory of situation awareness proposed in [3] is used with a Hidden Markov Model to represent this cognitive process. The first results in our simulation are promising but the study contains some limitations:

Firstly, the discretisation of the signals would lead to a degradation of the signification of the driving action associated. Therefore, a continuous version of HMM in which the observations are continuous signals may help better modeling these complex behaviors of human being.

Secondly, in the experiment, the first proposed hypothesis about the influence of the displayed messages at $4 s$ of TTC should be analyzed. It could be considered as a second task when the driver has to perceive and to decide to take into account this message. He can neglect the message, or perceives it lately. More details of this kind of study can be found in [15]. The second and the third hypotheses are also the complicated problems that are out of scope of this paper.

Thirdly, in the cross-validation method, although we can see the stability of the system, the disadvantage of this method is that some observations may never be selected in the validation sub-samples, whereas others may be selected more than once. In other words, validation subsets may overlap.

\section{CONCLUSION AND FUTURE WORKS}

This study helps to understand the behaviors of driver in a particular situation where a pedestrian appears in front of the vehicle. A model of awareness and unawareness of pedestrian as well as a recognition process have been proposed. The first validations showed promising results. A discussion on the limitations has been also highlighted. We hope this will encourage more investigation into the driver behaviors signals analysis in different situations.

In the future work, we will add the gaze and head tracking in order to better analyze these behaviors. The correlation between the driver's gaze direction to pedestrian and his reactions will be analyzed. Another model of DAP and DUP will be established and will be compared to the proposed model. Moreover, we will do a new experiment with some more scenarios, a distraction system integerated in the DUP simulation and with some more participants. Finally, a deeper inter-scenarios, inter-participant validation will be realized and a test in real driving conditions with our intelligent vehicle platform ${ }^{1}$ would be envisaged.

\section{ACKNOWLEDGMENT}

This work was carried out in the framework of the Labex MS2T, which was funded by the French Government, through the program " Investments for the future" managed by the National Agency for Research (Reference ANR-11-IDEX0004-02).

\footnotetext{
${ }^{1}$ Robotex, the national network of robotics platforms http: //equipex-robotex.fr/
}

\section{REFERENCES}

[1] P.G.A. Boy. The Handbook of Human-Machine Interaction: A HumanCentered Design Approach. Ashgate Publishing, Limited, 2012.

[2] S. Y. Cheng and M.M. Trivedi. Turn-intent analysis using body pose for intelligent driver assistance. Pervasive Computing, IEEE, 5(4):2837, Oct 2006.

[3] M. R. Endsley. Toward a theory of situation awareness in dynamic systems. Human Factors: The Journal of the Human Factors and Ergonomics Society, 37(1):32-64, 1995.

[4] Y. Fukagawa and K. Yamada. Estimating driver awareness of pedestrians from driving behavior based on a probabilistic model. In Intelligent Vehicles Symposium (IV), 2013 IEEE, pages 1155-1160, June 2013.

[5] V. Gadepally, A. Krishnamurthy, and U. Ozguner. A framework for estimating driver decisions near intersections. Intelligent Transportation Systems, IEEE Transactions on, 15(2):637-646, April 2014.

[6] T. Gandhi and M.M. Trivedi. Pedestrian protection systems: Issues, survey, and challenges. Intelligent Transportation Systems, IEEE Transactions on, 8(3):413-430, Sept 2007.

[7] J.C. Hayward, Pennsylvania Transportation, Traffic Safety Center, and National Research Council (U.S.). Highway Research Board. Meeting. Near Miss Determination Through Use of a Scale of Danger. Number 7115 in Report (Pennsylvania Transportation and Traffic Safety Center). Pennsylvania Transportation and Traffic Safety Center, The Pennsylvania State University, 1972.

[8] B. H. Juang and L. R. Rabiner. Hidden markov models for speech recognition. Technometrics, 33(3):251-272, 1991.

[9] C. Wang L. He, C. Zong. Driving intention recognition and behaviour prediction based on a double-layer hidden markov model. Journal of Zhejiang University SCIENCE C, 13(3):208-217, 2012.

[10] A. Liu and A. Pentland. Towards real-time recognition of driver intentions. In Intelligent Transportation System, 1997. ITSC '97., IEEE Conference on, pages 236-241, Nov 1997.

[11] J.C. McCall and M.M. Trivedi. Driver behavior and situation aware brake assistance for intelligent vehicles. Proceedings of the IEEE, 95(2):374-387, Feb 2007.

[12] J.C. McCall, D.P. Wipf, M.M. Trivedi, and B.D. Rao. Lane change intent analysis using robust operators and sparse bayesian learning. Intelligent Transportation Systems, IEEE Transactions on, 8(3):431440, Sept 2007.

[13] D. Mitrovic. Reliable method for driving events recognition. Intelligent Transportation Systems, IEEE Transactions on, 6(2):198-205, June 2005.

[14] K. Murphy. Hidden markov model (hmm) toolbox for matlab. Available: http://www.ai.mit.edu/ murphyk/Software/HMM/hmm.html.

[15] B. Metz N. Schomig. Three levels of situation awareness in driving with secondary tasks. Safety Science, 56(0):44 - 51, 2013. Situation Awareness and Safety.

[16] Oktal. Driving simulation engine scaner http://www.scanersimulation.com.

[17] L. Rabiner. A tutorial on hidden markov models and selected applications in speech recognition. Proceedings of the IEEE, 77(2):257-286, Feb 1989.

[18] T. Starner, A.P. Pentland, Massachusetts Institute of Technology. Media Laboratory. Vision, Modeling Group, and Massachusetts Institute of Technology. Perceptual Computing Section. Visual Recognition of American Sign Language Using Hidden Markov Models. M.I.T. Media Lab Vision and Modeling Group technical report. Perceptual Computing Section, Media Laboratory, Massachusetts Institute of Technology, 1995.

[19] M.M. Trivedi, T. Gandhi, and J. McCall. Looking-in and looking-out of a vehicle: Computer-vision-based enhanced vehicle safety. Intelligent Transportation Systems, IEEE Transactions on, 8(1):108-120, March 2007.

[20] WHO. World health organization, 2013. global status report on road safety supporting a decade of action.

[21] L. Yang, B.K. Widjaja, and R. Prasad. Application of hidden markov models for signature verification. Pattern Recognition, 28(2):161 $170,1995$.

[22] X. Zou. Modeling intersection driving behaviors: A hidden markovmodel approach-i. Journal of Transportation Research, pages pp.1623, 2006. 